\title{
Ratio of optic cup to optic disc
}

\section{In relation to axial length of eyeball and refraction}

\author{
A. TOMLINSON AND G. I. PHILLIPS \\ Department of Ophthalmology, University of Manchester
}

Armaly ( 1967 ) found that the ratio of the horizontal diameter of the optic cup to that of the optic disc, in three samples of first degree relatives, varied directly and similarly between parents and offsprings, and between siblings. The frequency distribution of the ratio did not differ in the three samples and was not influenced by age or sex. He concluded that the ratio was determined genetically in a multifactorial pattern. On the basis of his valuable and interesting observations, he also pointed out that cup-to-disc diameter ratio is not a good criterion of glaucomatous cupping, although inequality between the cups in two eyes may give rise to suspicion.

In the present survey, not only the horizontal diameters of the optic cup and disc have been measured, but also their areas, because the area ratio seemed likely to be a better parameter than diameter ratio. These ratios will be considered in relation to axial length and refraction of eyeballs.

\section{Subjects and method}

SUBJECTS

The survey was conducted on 75 adult students in the Department of Ophthalmic Optics, University of Manchester Institute of Science and Technology. Their ages were between 18 and 27 years; 26 were females and 49 males. Ocular tensions, measured by applanation tonometry, ranged from 10 to $19 \mathrm{~mm}$. $\mathrm{Hg}$. The results from only one eye of each subject were used for statistical purposes: this was randomly selected by the toss of a coin ( 36 right, 39 left).

\section{METHOD}

After applanation tonometry, the depth of the anterior chamber and corneal thickness were measured with the optical attachment for the Haag-Streit 900 slit lamp described by Jaeger (1952). The distance between the anterior surface of the lens and retina in each eye was next measured by an ultrasonographic technique similar to that described by Leary, Sorsby, Richards, and Chaston (1963) and by Storey (1968). The instrument used was a modified Ultrasonoscope mark $2 \mathrm{C}$ flaw detector. Ultrasonic measurement of the eye was followed by instillation of guttae ephedrine hydrochloride 4 per cent. and then by objective and subjective determinations of its refraction. Four photographs of each optic disc were then taken with the Zeiss fundus camera, the supplementary $2 x$ objective being used, and from them a stereoscopic pair of photographs was prepared.

A photographic method of determining the area of the cup and disc was chosen in preference to a direct ophthalmoscopic technique, mainly because it provided a permanent record for checking, if necessary, but also because it was difficult by ophthalmoscopy to obtain a static enough view of the disc for the time required to count graticule squares.

Great care was taken to obtain the maximum degree of standardization of the stereophotographs. Monochrome $\mathrm{FP}_{4}$ film was used throughout with a constant aperture setting and exposure time. 
To obtain a constant shift in fixation before the second photograph was taken, the following procedure was developed:

The back of the Zeiss model eye was removed, and the area of disc and macula covered by a small piece of graph paper. The model eye was then mounted on the chin rest of the retinal camera and the back moved away from the camera until it was in the position corresponding to the degree of ametropia of the subject to be photographed. The camera was focused on the back of the model eye and the cross-lines in the eye-piece were positioned over one of the intersections of the grid of the graph paper. The fixation reference mark within the camera was adjusted until it was in focus on the graph paper and until its end was positioned $5 \mathrm{~mm}$. from the intersection of the cross-lines in the eye-piece of the camera. The model eye was removed and the subject's head inserted into the head rest of the retinal camera. When the eye was not being photographed, the subject looked at a point on the wall behind the examiner and from this point he was told to look up, down, right, or left until the centre of his disc lay first at the intersection of the cross-lines in the eye-piece and then at the end of the fixation reference mark. Two photographs of the disc were taken at each of these two positions, and a reasonably constant displacement of successive photographs was thus obtained for subjects with differing refractive states.

The photographs obtained by this technique were mounted on card to form a stereopair and viewed by a variable prism stereoscope. The margin of the cup was marked and the area of cup and disc measured with a Stanley rule-form planimeter.

To test the validity of the standardization technique, seven stereopairs of photographs were taken of the eye of subject F.S.-2. The cup area: disc area ratio showed a mean ratio of 0.33 with an S.D. of o.or3, which we consider to indicate that the technique was satisfactorily standardized, i.e. we felt justified in basing the measurement of cup-to-disc area ratios on the results obtained from one stereopair. The area of cupping marked on the

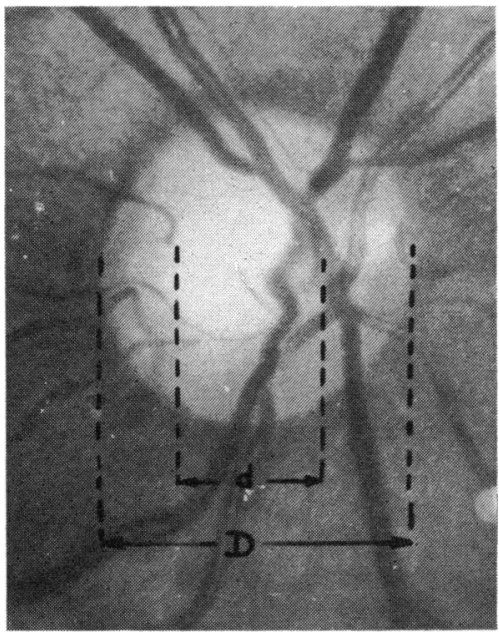
stereophotograph was, however, cross-checked ophthalmoscopically. The measurement of the horizontal diameters of the cup and optic disc was made with a finely graduated rule, as shown in the Figure. It is assumed that any difference between the magnification of the cup and that of the disc will be negligible, if present at all.

FIGURE Photograph of right optic disc taken with Zeiss fundus camera. Maximum horizontal diameter of cup and disc are indicated; from this, the cup-to-disc diameter ratio is obtained

\section{Results}

The values of the best sphere refraction, of axial length, and of the cup-to-disc area and diameter ratio were statistically analysed.

The distributions of axial length, cup-to-disc area ratios, and cup-to-disc diameter ratios were found not to differ significantly from the Gaussian by a $\chi$-squared test. The correlations between area ratio and axial length, and between diameter ratio and axial length, were calculated by a product moment method. The correlation coefficients (see Table) were significant at the 5 per cent. level.

The distribution of best sphere refraction was found to be non-Gaussian (as would be expected-see Sorsby, Benjamin, Davey, Sheridan, and Tanner, 1957-because of the tendency for the refractive powers of cornea and lens to vary in compensation for variations 
in axial length), and therefore the correlation between refraction and area ratio, refraction and diameter ratio, and between axial length and refraction had to be calculated by Spearman's rank correlation method. A significant correlation at the 5 per cent. level was found (see Table) between refraction and area ratio. (At the $0 \cdot 1$ per cent. level there was also a correlation between refraction and axial length).

Table Correlation of results

\begin{tabular}{|c|c|c|}
\hline Statistical test & Between & $\begin{array}{l}\text { Correlation } \\
\text { coefficient }\end{array}$ \\
\hline Product-Moment & $\begin{array}{l}\text { Area Ratio and Axial Length } \\
\text { Diameter Ratio and Axial Length }\end{array}$ & $\begin{array}{l}r=+0 \cdot 27^{*} \\
r=+0 \cdot 23^{*}\end{array}$ \\
\hline Rank Correlation & $\begin{array}{l}\text { Refraction and Area Ratio } \\
\text { Refraction and Diameter Ratio } \\
\text { Refraction and Axial Length }\end{array}$ & $\begin{array}{l}\rho=+0.23^{*} \\
\rho=+0.16 \\
\rho=+0.79 \dagger\end{array}$ \\
\hline
\end{tabular}

*Significant at 5 per cent. level

†Significant at $0 \cdot 1$ per cent. level

\section{Discussion}

These observations support the commonly held clinical impression that the more (axially) myopic an eye the larger the cup in relation to the whole disc and the more (axially) hypermetropic the eye the smaller the cup in relation to the whole disc.

The correlation between area ratio and axial lengths (see Table) is higher than that between diameter ratio and axial length.

Area ratio is significantly correlated with refraction whereas the correlation of diameter ratio with refraction fails to reach the 5 per cent. probability level. If the clinical impression be true that the optic disc is vertically oval, or at least not necessarily round, the GUP-DISC AREA RATIO is likely to be a better index of RELATIVE cupping than the HORIZONTAL DIAMETER RATIO and possibly a fairer index than the VERTIGAL DIAMETER RATIO.

The distribution of diameter ratios which we found was Gaussian, but this differs from the positive skew distribution reported by Armaly (1967), who found a 67 per cent. frequency below $0 \cdot 3$, whereas we found 67 per cent. below $0 \cdot 55$. This difference between the populations does not in our opinion detract from the validity of the observations on either. In our population, almost 50 per cent. were myopic (maximum $-6 \mathrm{D}$ ) which is probably a higher proportion than one would expect in a random sample of the population: it seems likely that students, especially of ophthalmic optics, may be to a limited extent self-selecting.

Stereoscopic fundus photographs obtained by the "successive shift" technique have been criticised by Donaldson (1964) and Allen (1964) mainly on the basis that the magnitude of the displacement of either the eye or the camera between the photographs greatly affects the apparent stereoscopic depth impression obtained. However, our aim was to relate area of cup to area of disc and not to measure depth of cup (an interesting property which we do not yet have the equipment to investigate): the stereoscopic view was merely an aid in precise indentification of the edge of the cup-a good example of its value is seen in the Figure, where without a stereoscopic view it would have been difficult to see the position of the edge of the cup in relation to the main vessels. Nevertheless it must be admitted that the constant-shift method we have used is not absolutely precise; but variations presumably affected eyes at random so that this source of inaccuracy does not detract from our observations or conclusions. 
We suggest that the explanation for the above findings probably depends on:

(a) The existence of a fairly standard number of nerve fibres of standard size with a standard amount of supporting tissue congregating at the disc in most individuals.

(b) The fact that the diameter and area of the disc itself vary with size of eyeball-just as corneal diameter, depth of anterior chamber, etc., vary between individuals.

(A similar mechanism is usually presumed to explain the stretching of the standard retina and choroid to line the abnormally long myopic eyeball, its frequent failure to stretch sufficiently being betrayed by the absence of retina and choroid over areas near the disc). Evidence in favour of $(b)$ above is given by Ishii ( $195 \mathrm{I}$ ), who found a positive correlation between the horizontal diameter of the optic disc and the sagittal diameter of the globe in a group of cadaver and enucleated eyes. An estimate of the absolute size of the disc is possible from a knowledge of the magnification of the refractive system of the eye in situ, and further support for this would be derived from the finding of a positive correlation between absolute size of the disc and the cup to disc ratio for the subjects used in this survey-a project which is planned. The absolute size of the optic disc may be important in its ability, or in the ability of its capillaries, to withstand raised intraocular pressure; we suspect that the larger the disc the lower is the intraocular pressure which will produce field loss, because of the clinical impression that low-tension glaucoma tends to occur in high axial myopes (even after allowance has been made for low ocular rigidity). It would also be interesting to do a family study to establish to what extent the strong hereditary influence on the cup-to-disc diameter ratio which Armaly (1967) found might produce its effect through inheritance of axial length (Sorsby, Sheridan, and Leary, 1962).

\section{Summary}

The ratio of horizontal diameter of optic cup to that of optic disc has already been established as genetically determined. In a series of 75 normal subjects with normal ocular tensions and axial lengths of eyeball between $21 \cdot 13$ and $27.84 \mathrm{~mm}$., the area of the optic cup relative to the total area of the optic disc (cup-to-disc area ratio) tended to be high when the eyeball was long and the refraction myopic, but low when the eyeball was short and the refraction hypermetropic; these observations were statistically significant at the 5 per cent. level. The likeliest explanation is that the absolute area of the optic disc probably varies with the size of eyeball, whereas the volume of nerve fibres congregated at the optic nerve head is fairly standard. To determine the importance of inheritance of axial length in the heredity of cup-to-disc diameter or area ratio will require a separate family study.

\section{References}

ALlEN, L. (1964) Amer. F. Ophthal., 57, 13

ARMALY, M. F. (1967) Arch. Ophthal. (Chicago), 78, 35

Donaldson, D. D. (1964) Trans. Amer. ophthal. Soc., 62, 429

ISHII, K. (1951) Acta Soc. ophthal. jap., 55, 242 (see Ophthal. Lit., I951, 5, No. 2270)

JAEger, W. (1952) v. Graefes Arch. Ophthal., 153, 120

LEARY, G. A., SORSBY, A., RICHARDS, M. J., and Chaston, J. (1963) Vision Res., 3, 487

SORSBY, A., BENJAMIN, B., DAVEY, J. B., SHERIDAN, M., and TANNER, J. M. (1957) "Emmetropia and its

Aberrations". Med. Res. Coun. Spec. Rep. Ser., No. 293

- Sheridan, M., and leary, G. A. (1962) "Refraction and its Components in Twins".

Med. Res. Coun. Spec. Rep. Ser., No. 303

storey, J. K. (1968) "Certain Ocular Dimensions". Ph.D. Thesis, University of Manchester 